\title{
La construcción legislativa uruguaya en economía social y solidaria en el marco de los gobiernos progresistas del Frente Amplio
}

The Uruguayan laws construction in social and solidarity economy in the framework of the progressive Governments of the Frente Amplio

A construção legislativa uruguaia em economia social e solidária no contexto dos governos progressistas da Frente Ampla

\section{Pablo Guerra* Sergio Reyes ${ }^{*}$}

Recibido: 4 de agosto de 2018 Aceptado: 3 de octubre de 2018 Publicado: 5 de abril de 2019

Cómo citar este artículo: Guerra, P. y Reyes, S. (2019). La construcción legislativa uruguaya en economía social y solidaria en el marco de los gobiernos progresistas del Frente Amplio. Cooperativismo \& Desarrollo, 27(1), 1-18. DOI: https://doi.org/10.16925/2382-4220.2019.01.05

Artículo de investigación. https://doi.org/10.16925/2382-4220.2019.01.05

* Universidad de la República Colonia, Montevideo, Uruguay. Correo electrónico: profecosol@yahoo.com ORCID: https://orcid.org/0000-0002-0154-3862

** Universidad de la República Colonia, Montevideo, Uruguay.

Correo electrónico: sreyes@reyeslavega.com.uy 


\section{Resumen}

En el presente artículo se analizan los aportes legislativos respecto a las temáticas del cooperativismo y de la economía social y solidaria (ESS), en el marco de los tres primeros gobiernos progresistas del Frente Amplio. Se destacan en el primer Gobierno de Tabaré Vázquez (2005-2010) la legislación en cooperativas sociales y la concreción de un viejo anhelo del movimiento cooperativo, esto es, una ley general del sistema cooperativo. Bajo el mandato de José Mujica (2010-2015) se destaca la creación del Fondo para el Desarrollo (Fondes), dirigido fundamentalmente a promover las experiencias autogestionarias. En el tercer mandato del FA (20152020), nuevamente bajo la presidencia de Vázquez, se formaliza el Fondes en ley y se avanza en la consideración de una legislación en ESs. Será de fundamental importancia comprender el contexto político-ideológico desde el cual se construyen estas normas. De esta manera, se pretende responder a las siguientes preguntas: ¿Qué razones explican el pasaje de un modelo meramente cooperativo hacia otro más amplio en términos de ESs? ¿Cómo fueron avanzando estos temas en la agenda programática del Frente Amplio? ¿Cuáles son las diversas orientaciones que pesan en la configuración de los proyectos de ley actualmente en discusión parlamentaria?

Palabras clave: cooperativismo, economía social y solidaria, legislación, políticas públicas, Uruguay.

\section{Abstract}

This article analyzes the legislative contributions regarding the themes of cooperativism and social and solidarity economy (ESS) in the framework of the first three progressive governments of the Frente Amplio. They emphasize in the first government of Tabaré Vázquez (2005-2010), the legislation in social cooperatives, and the concretion of an old yearning of the cooperative movement, that is, a general law of the cooperative system. Under the mandate of José Mujica (2010-2015), the creation of the Fondo para el Desarrollo (Fondes) is fundamentally aimed at promoting self-managed experiences. In the third mandate of the FA (2015-2020), again under the presidency of Vázquez, Fondes is formalized in law and continue the debate of legislation in ESs. It will be of fundamental importance to understand the political-ideological context from which these norms are constructed. It is thus that we try to answer the following questions: What reasons explain the passage from a merely cooperative model to a broader one in terms of ESS? How were these issues being advanced in the broader agenda of the Frente Amplio? What are the different orientations that weigh in the configuration of the bills currently in parliamentary debate?

Keywords: cooperatives, social and solidarity economy, laws, public policy, Uruguay.

\section{Resumo}

No presente artigo são analisadas as colaborações legislativas com respeito às temáticas do cooperativismo e da economia social e solidária (ess) no contexto dos três primeiros governos progressistas da coalizão Frente Ampla. No primeiro governo de Tabaré Vázquez (2005-2010) se destacam a legislação em cooperativas sociais e a concretização de um antigo desejo do movimento cooperativo, isto é, uma lei geral do sistema cooperativo. Sob o mandato de José Mujica (2010-2015) se destaca a criação do Fundo para o Desenvolvimento (Fondes), dirigido fundamentalmente a promover as experiências autogestionárias. No terceiro mandato do fa (2015-2020), novamente sob a presidência de Vázquez, formaliza-se o Fondes na lei e avança-se na consideração de uma legislação em ess. Será de fundamental importância compreender o contexto político-ideológico a partir do qual essas normas são construídas. Dessa maneira, pretende-se responder às seguintes perguntas: quais razões explicam a passagem de um modelo meramente cooperativo para outro mais amplo em termos de ess? Como esses temas foram avançando na agenda programática da Frente Ampla? Quais são as diversas orientações que pesam na configuração dos projetos de lei em discussão parlamentar atualmente? 
Palavras-chave: cooperativismo, economia social e solidária, legislação, políticas públicas, Uruguai.

\section{Introducción}

Al igual de lo que ocurrió en buena parte de la región sudamericana, en Uruguay hubo un proceso de legislación cooperativa que se inicia temprano en los comienzos y mediados del siglo xx. Para el caso nacional, debemos destacar la ley que crea la Cooperativa Nacional de Productores de Leche (Conaprole) en 1935; la Ley 10.008 de Cooperativas Agropecuarias (1941), y la Ley de "Sociedades Cooperativas" 10.761 de 1946, reguladora de las cooperativas de consumo y de las cooperativas de producción. Más atrás en el tiempo, las primeras leyes 3.948 y 3.949, del 19 de enero de 1912, se referían a las cajas de crédito rural —entidades estrechamente vinculadas en su funcionamiento al estatal Banco República-, y la Ley 6.192, del 16 de julio de 1918, a las sociedades de fomento rural, entidades no estrictamente cooperativas, pero que hoy podríamos llamar, sin duda, de la economía social y solidaria. En otro contexto histórico, la década de los sesenta también fue productiva en materia legislativa, de las que se destacan la Ley 13.481 (1966) sobre el régimen tributario de las cooperativas de producción o trabajo, la Ley N. ${ }^{0} 13.728$ (1968) sobre las cooperativas de vivienda, así como la Ley 14.019 —régimen tributario de las cooperativas de consumo—, y la Ley 13.988 — cooperativas de ahorro y crédito_, ambas de 1971. Luego de la recuperación democrática (1985), la legislación cooperativa fue apenas marginal, ${ }^{1}$ además de fragmentaria, a pesar de los intentos del movimiento cooperativo por impulsar una ley marco para todas las modalidades.

La hipótesis que manejamos en este artículo es que la asunción del partido Frente Amplio en el Gobierno nacional a partir del 2005 marca un nuevo hito en materia de legislación cooperativa, caracterizada por la concreción de una muy demandada ley marco del sistema cooperativo, por la creación de una nueva figura cooperativa -como es el caso de las cooperativas sociales-, así como por el nuevo protagonismo que adquirirá la economía social y solidaria y la economía autogestionaria, conceptos ambos que se irán abriendo paso, fundamentalmente, a través de la creación parlamentaria del Fondo para el Desarrollo (Fondes). Una segunda hipótesis que se maneja es que mientras los primeros dos instrumentos - ley marco y ley de cooperativas sociales - contaron con un amplio apoyo parlamentario que traspasó la barrera de los partidos, la inclusión de la economía social solidaria en los textos legislativos

1 Véanse la Ley 16.156 sobre personería jurídica de las cooperativas (1990), el Decreto 223/98: control estatal de las cooperativas (1998), y, sobre todo, la Ley 17.794 del 2004 sobre cooperativas de producción o trabajo asociado. 
contó con mayores reservas, habida cuenta de las diferencias conceptuales, así como ideológicas, que los diferentes actores políticos le asignan a términos que aún están en plena etapa de construcción y gestación de mayores consensos.

\section{Principales contribuciones legislativas en ESS en los tres primeros periodos de gobierno del Frente Amplio}

A poco de su inicio, el primer Gobierno del Frente Amplio aprobó la ley de cooperativas sociales (Ley 17.978 del 26 de junio del 2016), en el marco del denominado "Plan de emergencia", es decir, dentro de un conjunto de políticas sociales y de una novedad institucional: la creación del Ministerio de Desarrollo Social (MIDES). En la legislación uruguaya las cooperativas sociales son un subtipo de cooperativas de trabajo con algunas particularidades, a saber: 1. Por lo menos el $75 \%$ de sus integrantes deben ser calificados como vulnerables socialmente; 2. Las remuneraciones de sus miembros no pueden superar los salarios fijados por los convenios o laudos correspondientes a la rama de actividad en la que operen; 3. Los cargos directivos deben ser honorarios; 4. Están exoneradas de todo tributo nacional y de aportes a la seguridad social; 5. Pueden acceder a que sus servicios sean contratados de forma directa - sin procedimiento competitivo- por los organismos públicos, hasta el monto límite de la llamada licitación abreviada — hoy en día ese monto está radicado en uYu 8715 000, lo que equivale aproximadamente a USD 300 000-; y 4. Su formación debe ser autorizada por el Mides, organismo que también los controla durante su funcionamiento.

Asimismo, y como ya dijimos, habrá que esperar, por tanto, al primer Gobierno del Frente Amplio (2005-2010) para que el anhelo de una ley general se concretara en la actual Ley 18.407 del 24 de octubre del 2008, intitulada del "Sistema Cooperativo. Regulación general de su funcionamiento", ampliamente conocida como "Ley General de Cooperativas" (LGC). Si bien esta Ley es propiamente "cooperativa", instala por primera vez en un texto jurídico la voz "economía social y solidaria", la cual a la postre iría adquiriendo cada vez mayor protagonismo en las políticas públicas de los tres gobiernos analizados en este artículo. Con la creación del Instituto Nacional del Cooperativismo (Inacoop), se establece entre sus cometidos "impulsar el estudio y la investigación de otras formas de la economía social y solidaria y realizar propuestas sobre su alcance y regulación, de modo de favorecer la formación de un marco jurídico que facilite su desarrollo y promoción" (Ley 18407 del 2008). Justamente este artículo es el que ha activado el interés de Inacoop por legislar en la materia. 
Sin querer alejarnos del tema central de este artículo, es pertinente señalar que en lo concerniente a la regulación jurídica del cooperativismo se reconoce a esta Ley como un avance muy significativo, por cuanto su contenido es comprensivo y unificador de todas las cuestiones atinentes a las cooperativas, a saber: disposiciones programáticas y definiciones conceptuales, la constitución de las cooperativas y su reconocimiento jurídico, el control estatal, la relación jurídica con los asociados, el funcionamiento organizacional, el régimen económico-financiero, nuevos instrumentos para facilitar la capitalización, así como novedosas figuras en procura de una mejor puesta en práctica de la intercooperación. Al respecto, el reconocido doctrino uruguayo Siegbert Rippe señala:

La LGC constituye un instrumento jurídico adecuado para solucionar el problema de la anarquía de la legislación cooperativa nacional, a través de una ley general que reconoce la unidad sustantiva de las cooperativas en cuanto formas jurídicas regidas por iguales principios y similares reglas de organización y funcionamiento y que acepta a su vez las diversidades motivadas por las particularidades de los respectivos objetos sociales o las aconsejadas por la propia experiencia y realidad del cooperativismo nacional. (Rippe, 2009, p. 40).

Un segundo texto que menciona a la ESS es la Ley 19.337 del 20 de agosto del 2015, por la cual se crea el Fondo para el Desarrollo (Fondes). ${ }^{2}$ El artículo 13, en su literal A, señala como cometidos del Fondes-Inacoop: "Promover y apoyar el desarrollo de las distintas formas de la economía social y solidaria" (Ley 19337 del 2015). Al saber que estas dos legislaciones mencionan de forma explícita a la ESS sin definir sus alcances $y$, si se tiene en cuenta, además, que la primera de ellas conmina a Inacoop a formalizar un marco jurídico, se sobreentiende la necesidad de contar con una legislación específica, aspecto sobre el que volveremos más adelante.

Finalmente, la "economía social" también tiene en cuenta la Ley 19.472 del 23 de diciembre del 2016. En efecto, dicha Ley - por la que crea el Sistema Nacional de Transformación Productiva y Competitividad, con la finalidad de promover el

2 El Fondes es producto, en gran medida, del impulso del presidente José Mujica, quien propuso y logró que el Parlamento Nacional incluyese en el art. 40 de la Ley 18.716 del 24 de diciembre del 2010 (modificación de la Carta Orgánica del Banco República) la facultad del Poder Ejecutivo de destinar hasta un $30 \%$ de las ganancias anuales del Banco República (luego de impuestos y de ciertos resguardos patrimoniales) para el apoyo a emprendimientos autogestionarios. La reglamentación de este artículo y la creación del Fondes se llevo a cabo primero por decreto del Poder Ejecutivo (Decreto 341/011), hasta que en el 2015 se le introdujeron una serie de cambios y quedó incorporado con rango legal. 
desarrollo económico productivo e innovador, con sustentabilidad, equidad social y equilibrio ambiental y territorial- hace referencia a la "economía social", y la incluye como una de las destinatarias de los posibles apoyos (art. 1), al integrar el Sistema a través del Inacoop (art. 2) y, por último, al darle participación a los "emprendimientos de la economía social" en los Consejos Consultivos de Transformación Productiva y Competitividad (art. 9). Como se puede apreciar, en este caso la expresión utilizada es la de "economía social", sin el aditamento "y solidaria". Ni de la "Exposición de Motivos del Proyecto de Ley", ni de la discusión parlamentaria se desprende porqué se escoge esta opción terminológica, mas cabe de señalar que en el proyecto original ya presentaba esta redacción. Más allá de que algún legislador planteó el punto, este no mereció mayor atención, por lo menos así se desprende de las actas de la Comisión de Hacienda de la Cámara de Diputados, en las que el tema se trató en mayor medida. Quizás puede explicar la cuestión que se trató de un proyecto objeto de muchos cambios y de muchas horas de negociación y transacción política -dentro del propio partido de gobierno), en cuanto a aspectos medulares - por ejemplo, inicialmente el proyecto se intitulaba "Sistema Nacional de Competitividad", y en el Parlamento se cambió esa denominación por la de "Sistema Nacional de Transformación Productiva y Competitividad", y junto con el título fueron también modificados varios de los artículos-. Así, entonces, en ese contexto, aquello de batallar por lo de "economía social y solidaria" pudo ser algo secundario. También cabe notar que algunos actores políticos -incluso de izquierda-, en sus discursos se apegan más a la expresión "economía social" que a la de ESs. Esta interpretación no se desprende de las actas parlamentarias, sino que más bien se basa en nuestro conocimiento de la realidad extraparlamentaria. Sin perjuicio de lo antedicho, más adelante se verá con algo más de detalle el estado de la cuestión de la denominación y del contenido de la Ess.

Mientras esto ocurre en el plano legislativo, la ESS comienza a ganar espacios en materia de políticas públicas. Nótese cómo en el 2005 el Gobierno departamental de Canelones crea una Unidad de Economía Solidaria, tendencia que luego continúa el Gobierno departamental de Montevideo al crear una unidad específica de economía social y solidaria, inaugurada en el 2015. A nivel del Poder Ejecutivo, el Ministerio de Desarrollo Social optó por la voz "economía social" para una dirección ${ }^{3}$ que tiene a su cargo "diseñar e implementar políticas, programas y proyectos, que fortalezcan la integración al mundo del trabajo -en clave de economía social- de las personas en

3 Dicha repartición del Mides se denomina "Dirección Nacional de Economía Social e Integración Laboral". Tiene a su cargo, entre otras funciones, todo lo relativo al funcionamiento de las cooperativas sociales. 
condiciones de exclusión y/o vulnerabilidad social, económica y territorial" (Mides, 2017). Como se observa, el sesgo del Ministerio de Desarrollo Social con respecto a la población en situación de vulnerabilidad es uno de los aspectos que no ha contribuido en Uruguay al correcto discernimiento acerca de los alcances de la Ess como un formato económico que vaya más allá de los sectores excluidos.

\section{El interés legislativo sobre la ESS}

Respecto a la evolución que siguió la ESS en Uruguay, distinguimos tres etapas. La primera de ellas la ubicamos en la década de los noventa del siglo pasado. En esos años comienzan las actividades académicas a usar el término "economía solidaria". A partir de entonces, ciertos movimientos sociales, de iglesia y del movimiento cooperativo, utilizan el concepto como una forma de renovar el discurso y las prácticas basadas en la autogestión, la cooperación económica, la ayuda mutua o la democratización de los procesos económicos, en el marco de objetivos de más largo alcance como, por ejemplo, perseguir una sociedad más justa para algunos, o incluso de corte poscapitalista para otros. Entre los hitos de esta primera etapa deberíamos señalar los clubes de trueque y las primeras ferias de economía solidaria, ambos fenómenos alimentados por la profunda crisis socioeconómica que asomó sobre fines de la década de los noventa, para explotar definitivamente en el 2002. La segunda etapa se construye poco a poco de la mano de las numerosas delegaciones de movimientos sociales que participaron en la primera convocatoria en Porto Alegre al Foro Social Mundial (2001). Es el tiempo de las primeras instancias de coordinación de estos nuevos actores de la ESS. Se destaca en ese sentido el Espacio de Economía Solidaria (hoy inexistente), y luego la Coordinadora Nacional de Economía Solidaria (CNES). Cabe señalar que los actores tradicionales nucleados en la Confederación Uruguaya de Entidades Cooperativas (CUDECOOP) comienza en esta etapa también a compartir espacios de articulación con la CNES. La tercera etapa, mientras tanto, se caracteriza por la activación de las primeras políticas publicas dirigidas a algo más que el cooperativismo.

Es así que el interés legislativo nacional en las temáticas de la Ess forma parte de esta tercera etapa. Distinguimos aquí dos grandes momentos. Por un lado, la instalación de los términos "economía solidaria", "economía social" o "economía social y solidaria", indistintamente, en diferentes áreas de gobiernos tanto departamentales como en el Gobierno nacional. En un segundo momento esas voces llegan también a 
los dos textos legislativos mencionados. Ahora bien, ¿qué factores contribuyen a este interés? Destacamos en tal sentido tres explicaciones:

- Cierta incidencia política del movimiento de la economía solidaria.

- El mayor protagonismo de las tendencias legislativas en ESS en otros países de América Latina y Europa.

- El acercamiento doctrinario de la Ess con ciertos principios y valores políticos del gobierno de izquierda.

Con respecto a lo primero, si bien el movimiento de la economía solidaria es débil en Uruguay, sobre todo en el quinquenio 2005-2010, fue capaz de realizar diferentes actividades de incidencia política junto con los actores del movimiento cooperativo -un movimiento más fuerte y con mayor representatividad-, y hacia actores de varios gobiernos departamentales y del Gobierno nacional. Algunos indicadores de esta incidencia política fue la presencia de delegaciones de la economía solidaria en las instancias de las reuniones especializadas en cooperativismo del Mercosur (RECM); la presencia de autoridades ministeriales en ferias de economía solidaria; el programa de desayunos de trabajo de la tienda Ecosol, en los que participaron legisladores e integrantes del Poder Ejecutivo; la presencia de autoridades nacionales en varias instancias de trabajo de la CNES; entre otros. Este lobby es, además, el que habilitó el literal $\mathrm{M}$ de la Ley 18.407, por la cual se le encomienda al flamante Inacoop atender los asuntos de la Ess y avanzar hacia un marco regulatorio. Este articulado surge luego de una intensa discusión en la que algunos actores postulaban la necesidad de crear un instituto para toda la ESS y no solo para el cooperativismo. De esta manera, en lugar de un Inacoop, se planteó en la discusión parlamentaria la posibilidad de crear un INAESS O INACOOPESS. La presión de algunos sectores cooperativos que no estaban dispuestos a semejante apertura finalmente tuerce la balanza hacia un instituto de corte más tradicional. Sin embargo, con el propósito de atender los reclamos de quienes percibían la importancia de mayor apertura hacia la ESs se redacta ese literal del art. 187. Con relación a lo segundo, no cabe duda que la tendencia en otros países a regular el sector asociativo -más allá de los paradigmas tradicionales regulatorios propios de cooperativas y mutuales-, jugó un rol importante. Los dirigentes nacionales del cooperativismo observan que la ESS ya no es solo un movimiento social con mayor o menor peso en diferentes países, sino además una realidad que comienza a cuajar en legislaciones de países - sobre todo de América Latina y Europa-. Comienzan a percibir, además, que la Ess no es algo distinto al cooperativismo, sino una suerte de paraguas que los incluye. Se 
trata, finalmente, no solo de una mera inclusión, sino de una en la que el cooperativismo forma parte sustancial y especialmente protagónica. Estos aspectos serán de fundamental importancia para apalancar el interés del movimiento cooperativo en estas materias. Debemos destacar que eventos de la Alianza Cooperativa Internacional ( $\mathrm{ACl}$ ) -como, por ejemplo, el caso de las cumbres regionales- al promover actividades en el marco del paradigma de la Ess contribuyeron enormemente al respecto. También el hecho que algunos de los dirigentes de la Red Internacional de Promoción de la Economía Social Solidaria (RIPESS) pertenezcan al movimiento cooperativo.

La tercera fuente explicativa no es de menor importancia. Muchos de los promotores de la economía solidaria en Uruguay provenían de diferentes expresiones de la izquierda nacional que confluyen en el partido de gobierno, esto es, el Frente Amplio. Había, además, una natural coincidencia entre el discurso de la economía solidaria a favor de una economía más justa y solidaria -incluso de corte anticapitalista en algunos casos, y poscapitalista en otros - con los discursos históricos de la izquierda nacional. Todos estos elementos llevan a que, primero en el "Programa de Gobierno de Montevideo", y luego en el "Programa de Gobierno nacional" se incluyeran sendos pasajes referidos a la ESS. De esta manera, en el VI Congreso Extraordinario del Frente Amplio (2013), y luego de muchas discusiones, se acuerda la redacción de las "Bases programáticas". Lo referente a la Ess tiene lugar en el capítulo sobre el desarrollo. Allí se define la ESS, se mencionan los logros del Gobierno en estas materias y se postula la necesidad de contar con un plan de desarrollo de la ESs, entre otros asuntos (Frente Amplio, 2013).

\section{Estado de situación respecto a una legislación específica en ess}

La génesis de una eventual ley en Ess se remonta al esfuerzo realizado por algunos actores específicos del movimiento de la economía solidaria en Uruguay, entre el 2012 y el 2015, etapa en la que hubo una serie de eventos académicos y gremiales que tuvieron como propósito instalar el debate sobre la pertinencia de una ley específica. Un nuevo impulso tiene lugar cuando el Instituto Nacional del Cooperativismo (INACOOP) formaliza una comisión de trabajo en el 2014. Esa comisión, luego de algunos meses de reuniones, presenta un primer borrador de Ante Proyecto de Ley en la sesión del Consejo Consultivo de Inacoop de febrero del 2015. Allí, el anteproyecto fue valorado positivamente por todos los actores cooperativos, aunque surgieron dudas sobre la 
oportunidad -otros temas dominaban la agenda del cooperativismo y de INACOOP en ese momento-. También algunas federaciones pidieron tiempo para discutirlo en su interior. Por diferentes motivos -entre ellos el protagonismo que adquirió la ley que conforma el Fondes, ahora ya administrado por INACOOP-, el tema queda encajonado hasta el 2017, año en el que Cudecoop convoca a un desayuno de trabajo para relanzar el tema. En un salón nutrido de representantes del cooperativismo, de las redes de economía solidaria e integrantes de Inacoop, los presentes repasan el mismo borrador presentado dos años atrás y acuerdan revitalizar su discusión para presentar formalmente el proyecto en el Parlamento. ${ }^{4}$ Con el fin de distinguirlo del siguiente proyecto, lo denominamos "Proyecto 1".

En efecto, mientras eso ocurre, algunos diputados oficialistas presentan en marzo del 2017 un nuevo proyecto de ley de ESs, pero esta vez enfocado en las compras públicas. Este se titula "Promoción de la economía social y solidaria a través de las compras estatales". A este lo denominamos "Proyecto 2".

Como es notorio, no había mayores coordinaciones entre el trabajo realizado por Inacoop y el realizado por estos diputados, más allá de que el Proyecto 2 utiliza para su redacción sendos pasajes del Proyecto 1. Sin duda, una de las lecciones de este proceso es que se debió coordinar mejor esos asuntos, de manera que fuera posible establecerse una estrategia más elaborada a nivel de gobierno. Nótese que el Proyecto 1 presenta un capítulo de compras públicas, en tanto el Proyecto 2 presenta articulados que estaban presentes en el texto anterior. Lo cierto es que en vez de una estrategia conjunta, tenemos un escenario con un proyecto de impulso de la Ess por medio de las compras públicas y enviado a estudio por las comisiones parlamentarias, y otro anteproyecto más genérico al interior de Inacoop, sobre el que se sabe que sobre finales del año pasado se envió oficialmente por este instituto al presidente de la Comisión Especial de Cooperativismo de la Cámara de Diputados, pero estrictamente aún no tiene estado parlamentario (los proyectos de leyes pueden generarse por iniciativa de cualquier legislador o por el Poder Ejecutivo, de acuerdo con el art. 133 de la Constitución). Por encima de esos desajustes, como se trata en el siguiente capítulo, existen entre los proyectos concepciones y alcances similares acerca de lo que se entiende por ess, aunque también existen matices que podrían mostrar algunos de esos nudos gordianos en materia conceptual.

4 El único agregado al documento presentado fue la necesidad de incorporar de forma explícita a las mutuales de salud como actores de la ESS. El borrador presentado no las incluyó por diferencias entre integrantes de la Comisión (en la que, probablemente, estaba presente la disputa entre una concepción sectorial y otra más autogestionada acerca de lo que se entiende por ESS). Sin embargo, en un marco más amplio la discusión fue favorable para que un subsector que nuclea a cuatro entidades mutuales con decenas de miles de asociados también pudieran formar parte de la ley. 


\section{La ESS: un concepto aún en construcción}

Sobre las diferentes nociones de economía social y economía solidaria, destacamos las siguientes.

Con respecto al uso de "economía social" dos principales orientaciones han predominado en los últimos años, a saber:

- Orientación clásica. Los académicos y actores sociales más influidos por la literatura francófona adoptan la idea de la economía social tal como fue concebida a partir de la década de los setenta del siglo xx por los europeos. Esta lectura fue casi marginal hasta la década de los noventa, pues hasta entonces fue muy raro que se integraran las mutuales y las asociaciones de manera conceptual a un mismo sector junto con las cooperativas, estas sí reconocidas legalmente desde temprano por parte de todos los países latinoamericanos. Un ejemplo de esta tendencia en materia legislativa es la que se expresa en la Constitución Bolivariana de Venezuela. El artículo 184 se refiere a la promoción de la "economía social", y menciona el caso de "cooperativas, cajas de ahorro, mutuales y otras formas asociativas" (Venezuela, 1999).

- Orientación paternalista. Sobre finales de la década de los noventa surge, fundamentalmente en el campo de las políticas públicas, una visión más bien reduccionista y paternalista sobre la economía social, esto es, entenderla como un conjunto de políticas dirigidas a incluir socialmente a los sectores más vulnerables de la población. De esta manera se crean diversas políticas de subsidios a la pobreza y transferencias monetarias condicionadas a cierto emprendurismo individual o colectivo muy fuertemente atado a las iniciativas del Estado benefactor. El surgimiento de la Dirección de Economía Social en los ministerios de desarrollo social tanto de Argentina como del Uruguay son ejemplos de esta orientación. Según Coraggio, para el caso Argentino,

Se va institucionalizando la 'política social de economía social' focalizada en los sectores pobres y/o con problemas de empleo y dirigida a su inclusión en el mercado [] En todo caso, se evidencia que estos programas 
ven la Economía Social no como una alternativa a las empresas de capital o públicas, sino como una opción para suplir, al menos parcialmente, la insuficiencia de las políticas de inversión y generación de empleos por esos actores. (2011, p. 3-6).

Con respecto al uso del término "economía solidaria" en Guerra (2012), se distinguen dos grandes orientaciones que influyen en las políticas públicas y las legislaciones nacionales:

- Orientación sectorial. Hace referencia a la economía solidaria como un tercer sector de la economía, diferente al capitalista y al estatal, de carácter asociacionista y regido por ciertos valores como, por ejemplo, la cooperación y la ayuda mutua. Se trata, sin duda, de un equivalente a la economía social, aunque con una mayor precisión conceptual, en tanto el comportamiento solidario es más distintivo que la mera recurrencia a lo "social". Colombia representa bien esta orientación a partir de las elaboraciones sobre comienzos de la década de los noventa. Según el Dansocial, integran el sector solidario tres dimensiones: el sector cooperativo y mutual; otras formas de organizaciones comunitarias; y organizaciones sociales que realizan actividades solidarias de desarrollo:

Si bien la economía solidaria es una construcción normativa colombiana, mantiene referentes organizacionales de origen cooperativo y, en su extensión, abre espacios para prácticas solidarias afines, en alguna medida, a las desarrolladas en el ámbito europeo con la economía social. (Álvarez, 2007, p. 104).

- Orientación autogestionaria. La economía solidaria se limita a numerosas experiencias colectivas en las que no se reproduce la división entre empleado y empleador, o bien,

Conjuntos de emprendimientos económicos asociativos donde (i) el trabajo, (ii) los resultados económicos, (iii) la propiedad de los medios, (iv) el poder de decisión y (v) los conocimientos acerca de su funcionamiento son compartidos solidariamente por todos los que de ellos participan. (Cruz, 2011, p. 14). 
El mapeo de economía solidaria de Brasil, por ejemplo, excluye a las cooperativas o emprendimientos asociativos que contratan mano de obra para cumplir con sus propósitos. El trabajo que desarrolló la Senaes en Brasil responde a este modelo.

Además de esas dos grandes orientaciones, en los últimos años han surgido otras con mayor o menor intensidad. Por ejemplo, en varios de los movimientos alternativos de Europa y Latinoamérica es más usual la recurrencia a la voz "economía solidaria" para hacer hincapié en una visión más transformadora respecto a la mera "economía social". Desde este punto de vista, no se trata solo de fomentar el asociativismo con formas democráticas, sino de transformar con mayor contundencia las diferentes fases de la economía al promover acciones alternativas en el campo del consumo, las finanzas, la comercialización y la producción con énfasis en las dimensiones sociales y ambientales. La economía solidaria vendría a ser el sector más militante y transformador de la economía social, así como el más innovador en temáticas relacionadas con valores como, por ejemplo, la transparencia, la equidad de género o el cuidado medioambiental, por citar solo tres aspectos que definen a muchas experiencias de lo que algunos autores denominan "la otra economía".

La pregunta que asoma entonces para el caso nacional es cuál de estas nociones y orientaciones es la que prima en los textos que actualmente se discuten. Veamos esos asuntos con atención en los dos proyectos o anteproyectos que hoy están en discusión en Uruguay.

En primer lugar se encuentra el anteproyecto elaborado por la comisión de trabajo impulsada por Inacoop, la cual, en su artículo 3, define a la ESs en los siguientes términos:

Se entiende por Economía Social y Solidaria al sistema socioeconómico, cultural y ambiental, conformado por el conjunto de organizaciones y redes que desarrollan fines colectivos o sociales, caracterizado por las prácticas solidarias, asociativas, cooperativas y de ayuda mutua, la participación democrática en la toma de decisiones, la autonomía de la gestión, la primacía del ser humano y del bien común sobre el capital, la equidad social y de género, el respeto a la naturaleza, la diversidad cultural y el desarrollo local, con el objetivo de satisfacer las necesidades de sus integrantes o de la comunidad en procura del desarrollo humano y la democratización de la economía a partir de la producción de bienes y servicios, su distribución, circulación, comercialización, financiamiento y consumo justo y responsable. (Anteproyecto de Ley de ESS, 2015). 
Lo primero que asoma en esta definición es que se opta por la voz "economía social y solidaria", una solución política que une a quienes en Uruguay han preferido una u otra por diferentes razones. En segundo lugar, surge de la definición una orientación de corte sectorial - de hecho este artículo comienza con pasajes muy similares a la Ley 454 de Colombia que, como se señaló, es claramente de perfil sectorial-. Dadas las tantas y supuestas características que deberían tener estas entidades y el tenor de algunas de ellas, también podemos decir que existe un perfilamiento hacia las orientaciones más transformadoras propias de las corrientes que apuestan por "otra economía". Esta cita de supuestas características establece un primer punto de discusión del Proyecto de ley: ¿Es condición para formar parte del sector cumplir con todas esas características? ¿Qué sucede con las cooperativas que, por ejemplo, no establecen políticas de equidad de género, de protección ambiental o de consumo responsable? Finalmente, ¿cómo sabemos si realmente se cumplen esas características? A nuestra manera de ver, la ley debería explicitar una política nacional de promoción de la economía solidaria de manera que se trabaje con aquellas organizaciones que aún no han desarrollado acciones en las dimensiones sociales y ambientales señaladas. Debería, además, establecer la obligatoriedad de mecanismos del tipo triple balance, de manera que todas las organizaciones visibilicen sus aportes distintivos y así aseguren que cumplen con los valores que promueve la ESS. Esto ocurre en varias entidades de la ESS a nivel de la sociedad civil en diferentes países, aunque en Uruguay son instrumentos aún muy marginales. En definitiva, un primer nudo gordiano que advertimos de este proyecto es que al intentar posicionarse no solo en el plano meramente sectorial, sino además al incorporar nociones propias de las orientaciones más transformadoras, la ley puede caer en la tentación de levantar la vara con respecto a los principios y los valores que deberían guiar a los emprendimientos de la ESS. Por ejemplo, no bastaría ser una mera cooperativa o incluso una mera empresa autogestionada para ser parte de la ESS; además, estos emprendimientos deberían incorporar políticas específicas en el plano ambiental o de otras prácticas consideradas relevantes para la economía solidaria, como, por ejemplo, el consumo responsable, el comercio justo o el manejo ético de las finanzas.

El Proyecto 2, por su parte, tiene como objetivo reservar una cuota del 30\% de las compras estatales para emprendimientos de la ESS que formen parte de las mipymes -en el marco de ese proyecto, empresas nacionales con hasta 99 trabajadores-. Define a la ESS con base en -casi textualmente- el primer párrafo del Proyecto 1, lo que nos lleva a pensar que sus autores tomaron como base lo trabajado por la Comisión convocada por Inacoop. En su artículo 3 señala: 
Se entiende por Economía Social y Solidaria al sistema socioeconómico y cultural, conformado por el conjunto de organizaciones y redes que desarrollan fines colectivos o sociales, caracterizado por las prácticas solidarias, asociativas, cooperativas y de ayuda mutua. (Proyecto de Ley sobre promoción de la economía social y solidaria a través de las compras estatales del 2017).

Resulta llamativo que lo único que diferencia esta definición de la anterior es que al referirse al "sistema" prescinden de la voz "ambiental", un dato preocupante por el valor que los temas ecológicos han adquirido, sobre todo en el subsector más militante e innovador de la ess de los últimos años.

La orientación que define a este Proyecto de ley, mientras tanto, más allá de esta ligera diferencia en la definición respecto al Proyecto 1, parecería ser de corte autogestionaria. ${ }^{5}$ Esa orientación, a nuestro parecer, viene dada tanto por la exposición de motivos del proyecto -en la que se alude de forma directa a la necesidad de apoyar las empresas autogestionadas-, como por el tenor de los artículos 5 y 7 , fundamentalmente. Señala el artículo 5:

Los emprendimientos de la Economía Social y Solidaria se caracterizan por desarrollar su actividad de producción o provisión de servicios de modo tal que los medios de producción y la organización del trabajo responden a la propiedad colectiva, la gestión participativa, la autogestión y la solidaridad, con el objetivo de satisfacer las necesidades de sus integrantes en procura del desarrollo humano y la democratización de la economía a partir de la producción de bienes y servicios, su distribución, circulación, comercialización, financiamiento y consumo justo y responsable. [Cursivas añadidas] (Proyecto de Ley sobre promoción de la economía social y solidaria a través de las compras estatales del 2017).

5 La Federación de Cooperativas de Producción del Uruguay (FCPU) también interpretó que podía haber una orientación autogestionaria en este texto por la redacción dada a los primeros artículos del proyecto, sobre todo al artículo 5 , cuando señala que la propiedad de los medios y la organización del trabajo respondan a la propiedad colectiva; además del artículo 7 . Al respecto, la FCPU llama la atención para que esta redacción no se reduzca a los emprendimientos autogestionados, sino a toda la ESS en consonancia con el Proyecto 1. También tiene una posición crítica respecto a considerar solo a las mipymes y deja por fuera a empresas de la ESS con más de 99 trabajadores. Véase al respecto FCPU (2017). 
Esta redacción podría interpretarse en un sentido excluyente con respecto a aquellas entidades de la ESs en las que el trabajo no lo aportan sus socios, como en el caso emblemático de las cooperativas de usuarios.

Esta idea se refuerza en el artículo 7 sobre la contratación de trabajadores de manera extraordinaria. Allí se señala:

Los emprendimientos podrán contratar trabajo asalariado adicional sobre su nómina de socios con destino a tareas o exigencias que puedan considerarse extraordinarias o transitorias, siempre y cuando las mismas no desvirtúen el tipo de emprendimiento ni los principios orientadores de la presente ley. (Proyecto de Ley sobre promoción de la economía social y solidaria a través de las compras estatales del 2017).

Lo primero que cabe señalar al respecto es que se trata de una redacción confusa. Es claro que las cooperativas de usuarios contratan personal en relación de dependencia para garantizar el servicio a sus asociados. También es evidente que las cooperativas de trabajadores lo hacen, aunque con un tope respecto al número de socios trabajadores (20\% según la Ley 18.407). Este artículo, por tanto, no parece ir dirigido a cooperativas de usuarios, sino a las de trabajadores. Si eso fuera así, entonces el Proyecto 2 se muestra potencialmente peligroso para los valores autogestionados, pues estaría flexibilizando la norma del 20 \% prevista por el artículo 100 de la LGC.

En resumen, si bien en el contexto nacional surgieron debates acerca de si la ESS debía restringirse al campo autogestionario o ampliarse a todo el abanico sectorial -la discusión del Fondes y la impronta personal del expresidente Mujica tuvo mucho que ver en estos asuntos-, lo cierto es que el texto elaborado por la comisión convocada por Inacoop tiene un enfoque sectorial amplio (que incluye desde las grandes cooperativas de usuarios hasta las redes de pequeños productores artesanales), de modo que si bien el proyecto de ley elaborado por los diputados oficialistas recoge muchos pasajes de ese texto, presenta un perfil más autogestionado.

Consideramos que lo más importante en materia de avances es que hoy existe en Uruguay interés por regular esos asuntos. Los dos proyectos presentados tienen puntos en común que se cruzan de forma notoria, de manera que hace falta una estrategia consensuada. Lo más sensato sería elaborar un solo proyecto de ley de promoción de la Ess que incluyera un capítulo sobre compras estatales. En este será necesario trabajar mejor algunos aspectos que se refieren a las distancias que existen entre lo ideal y lo que ocurre realmente en las organizaciones del sector, y sobre cómo una política en la materia puede ayudar en ese sentido. 


\section{Conclusiones}

Uruguay forma parte de un grupo de países que desde hace algunos años introduce de manera progresiva el enfoque de la economía social y solidaria en sus políticas públicas y en sus legislaciones. Como en todos los casos, se trata de dar cabida a una nueva generación de actores socioeconómicos para los que las meras políticas y legislaciones en materia cooperativa y mutual, con un origen muy remoto, resultaban insuficientes.

Como hemos expuesto en este artículo, los tres gobiernos del Frente Amplio, con mayorías parlamentarias, han permitido dar nuevos bríos a la legislación cooperativa (Ley 17798; Ley 18407, con sus sucesivas reformas parciales), así como abrir las puertas a la autogestión y a la Ess como objeto de tratamiento jurídico. Mientras la autogestión se canalizó por medio de la creación del Fondes y su posterior regulación jurídica por ley (Ley 19337), la ESS comienza a adquirir rango jurídico con referencias a ella en la LGC, a lo que sigue la Ley 19337 y la Ley 19472. Ha pasado entonces a ser una prioridad del Inacoop legislar en concreto sobre la ESS, asunto que la llevara a presentar un anteproyecto de ley al Parlamento. Como hemos podido advertir, su discusión se vio demorada a causa de otro proyecto presentado en paralelo por un grupo de diputados oficialistas. Más allá de los problemas de coordinación estratégica que revelan estos hechos, quedan expuestas también algunas diferencias de orientación acerca de qué alcance le pretenden dar a la ESS lo diferentes actores sociales y políticos.

En fin, la construcción legislativa en ESs durante estos años de gobierno progresista fue de importancia tanto para el movimiento cooperativo como para el movimiento de la economía solidaria y de la autogestión. De concretarse una ley marco para la ESS, se estaría dando un nuevo paso para el impulso de las diferentes fórmulas asociativas bajo un paradigma conjunto y amplio, lo que sin duda contribuiría a la tarea de democratizar la economía.

\section{Referencias}

Álvarez, J. F. (2007, enero-mayo). El tercer sector y la economía solidaria. Revista Cooperativismo y Desarrollo, (90), 95-105.

Anteproyecto de Ley de ESS. [Comisión de Trabajo por Ley de ESS]. Montevideo, Inacoop, 2015.

Constitución de la República Bolivariana de Venezuela [Const]. (Venezuela). Recuperado de http://pdba.georgetown.edu/constitutions/venezuela/ven1999.html 
La construcción legislativa uruguaya en economía social y solidaria

Coraggio, J. L., Arancibia, M. I. y Deux, M. V. (2010). Guía para el mapeo y relevamiento de la economía popular y solidaria en América Latina y el Caribe. Perú: Gresp-Rippes.

Cruz, A. (2011). La acumulación solidaria. Revista de Estudios Cooperativos, 16(1), 12-36.

FCPU. (2017). Nota a la Comisión Especial de Marco Cooperativo de la Cámara de Representantes. Manuscrito inédito. Montevideo.

Frente Amplio. (2013). Bases programáticas 2015-2020. Frenteamplio.uy. Recuperado de https:// frenteamplio.uy/nuestra-voz/programa-de-gobierno-2015-2019

Guerra, P. (2012). Las legislaciones sobre economía social y solidaria (Documento de Trabajo N. 4, Facultad de Derecho, Universidad de la República).

Ley 18407 del 2008. Montevideo. Noviembre 14 del 2008.

Ley 19337 del 2015. Creacion del Fondo para el Desarrollo (Fondes). Agosto 20 del 2015. Recuperado de https://www.impo.com.uy/bases/leyes/19337-2015

Ley 19.472 del 2016. IMPO. Recuperado de https://www.impo.com.uy/bases/leyes/19472-2016

Ministerio de Desarrollo Social-Mides (2017). Dirección Nacional de Economía Social e Integración Laboral. Recuperado de http://www.mides.gub.uy/58454/direccion-nacional -de-economia-social-e-integracion-laboral-del-mides

Proyecto de Ley sobre promoción de la economía social y solidaria a través de las compras estatales [Comisión de Hacienda]. (2017). Montevideo, Repartido N. 650, Poder Legislativo.

Rippe, S. (2009). Innovaciones introducidas en la legislación cooperativa de Uruguay por la nueva Ley General de Cooperativas. Boletín de la AIDc. Bilbao: Deusto Publicaciones. 